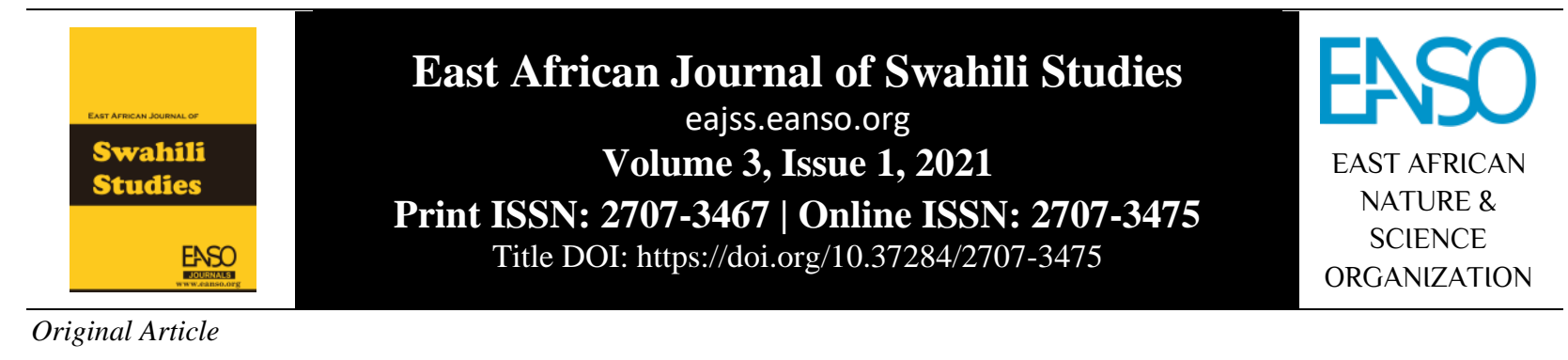

\title{
Athari za Kimofosintaksia za Ngeli za Luganda katika Matumizi ya Kiswahili Sanifu Miongoni mwa Wanafunzi Shuleni, Uganda
}

\author{
Mulei Martin ${ }^{1},{ }^{*}$ Dkt. Debora Nanyama Ph.D ${ }^{1}$ na Dkt. Beverlyne Ambuyo, Ph.D ${ }^{1}$ \\ ${ }^{1}$ Chuo Kikuu cha Kabale School, S. L. P. 317, Kampala, Uganda. \\ *Barua pepe ya mawasiliano: muleim1@gmail.com
}

DOI ya Nakala: https://doi.org/10.37284/eajss.3.1.331

\section{Tarehe ya Uchapishaji: IKISIRI}

19 Mei 2021 Makala hii inaonesha athari za kimofosintaksia za ngeli za Luganda katika

Maneno Muhimu:

Mofosintaksia,

Kanuni,

Athari,

Upatanishi wa Kisarufi,

Lugha ya Kwanza,

Umilikifu Na Unganifu,

Uhamishaji,

Uchopekaji,

Ujumlishaji,

Ubadilishanaji. matumizi ya Kiswahili sanifu. Makala hii inatokana na kuwa, Luganda na Kiswahili huainisha ngeli kutumia mfumo mmoja na matumizi yake kuzingatia sheria za kuonyesha upatanishi wa kisarufi wa nomino na maneno mengine katika tungo kimofosintaksia. Uwiano na tofauti za kanuni za matumizi ya vipashio vya kimofosintaksia baina ya lugha asili na Kiswahili sanifu umeripotiwa kuingiliana na kuathiri matumizi ya Kiswahili sanifu. Utafiti huu ulifanywa baada ya kubaini kuwa maandishi na mazungumzo ya wanafunzi ambao lugha yao ya kwanza ni Luganda na ambao wanajifunza Kiswahili kama lugha ya pili ulikiuka utaratibu wa kisarufi wa matumizi ya Kiswahili sanifu. Data ilitokana na uchanganuzi wa maandishi na mazungumzo ya wanafunzi 117, usaili na mijadala ya walimu 30 kutoka shule za upili 15 teule, wilayani Kampala, Uganda. Utafiti huu uliongozwa na mihimili ya sarufi bia na upatanifu wa nadharia ya Umilikifu na unganifu (Chomsky, 1981) ambayo huonesha ubia wa lugha na hali ya vipashio fulani vya kisarufi kutawala vipashio vingine katika tungo. Matokeo ya tathmini ya kanuni za matumizi ya kipashio cha kimofosintaksia cha ngeli za Luganda katika matumizi ya Kiswahili sanifu yalidhihirisha athari za; uhamishaji wa maumbo, mofofonolojia, ubebaji wa viambishi vya ngeli tofauti, uchopekaji wa viambishi, ubadilishanaji wa viambishi ngeli, uchanganyaji wa maumbo na ujumlishaji wa viambishi vya msingi vya ngeli ya Luganda katika matumizi ya Kiswahili sanifu. Makala hii inatarajiwa kuwasaidia walimu na wanafunzi kutambua athari za lugha ya kwanza katika matumizi ya Kiswahili sanifu, kuweka mikakati ya kuondokana na athari hizi kujenga matumizi bora ya Kiswahili sanifu.

10 This work is licensed under a Creative Commons Attribution 4.0 International License. 


\section{APA CITATION}

Martin, M., Nanyama, D., \& Ambuyo, B. (2021). Athari za Kimofosintaksia za Ngeli za Luganda katika Matumizi ya Kiswahili Sanifu Miongoni mwa Wanafunzi Shuleni, Uganda. East African Journal of Swahili Studies, 3(1), 116-127. https://doi.org/10.37284/eajss.3.1.331

\section{CHICAGO CITATION}

Martin, Mulei, Debora Nanyama, and Beverlyne Ambuyo. 2021. "Athari za Kimofosintaksia za Ngeli za Luganda katika Matumizi ya Kiswahili Sanifu Miongoni mwa Wanafunzi Shuleni, Uganda”. East African Journal of Swahili Studies 3(1), 116127. https://doi.org/10.37284/eajss.3.1.331.

\section{HARVARD CITATION}

Martin, M., Nanyama, D. and Ambuyo, B. (2021) “Athari za Kimofosintaksia za Ngeli za Luganda katika Matumizi ya Kiswahili Sanifu Miongoni mwa Wanafunzi Shuleni, Uganda”, East African Journal of Swahili Studies, 3(1), pp. 116-127. doi: 10.37284/eajss.3.1.331.

\section{IEEE CITATION}

M. Martin, D. Nanyama, and B. Ambuyo, “Athari za Kimofosintaksia za Ngeli za Luganda katika Matumizi ya Kiswahili Sanifu Miongoni mwa Wanafunzi Shuleni, Uganda”, EAJSS, vol. 3, no. 1, pp. 116-127, May. 2021.

\section{MLA CITATION}

Martin, Mulei, Debora Nanyama, and Beverlyne Ambuyo. "Athari za Kimofosintaksia za Ngeli za Luganda katika Matumizi ya Kiswahili Sanifu Miongoni mwa Wanafunzi Shuleni, Uganda”. East African Journal of Swahili Studies, Vol. 3, no. 1, May 2021, pp. 116-127, doi:10.37284/eajss.3.1.331.

\section{UTANGULIZI}

Athari za vipashio vya kiisimu vya lugha ambazo zina mtagusano na ukuruba wa muda mrefu katika matumizi, huweza kujitokeza kutokana na uhamishaji wa vipashio, viwakilishi vya vipashio vya kisarufi pamoja na sheria zinazoongoza matumizi ya vipashio hivyo vya kiisimu vya lugha ya kwanza kwa lugha ya pili. Athari hizi huweza kujitokeza katika upande mmoja au pande mbili ambapo L1 (lugha ya kwanza) huweza kuathiri L2 (Lugha ya pili) au kinyime. Athari za aina hii huweza kujitokweza katika maandishi au mazungumzo kimofolojia, kisintaksia, kisemantiki au kipragmatiki. Athari hizi hutokana na viwango vya umilisi wa lugha alionao mwanafunzi wa lugha baina L1 na L2. Aidha, athari hizi huweza pia kutokana na mikakati anayotumia mjifunzaji kurahisisha ujifunzaji na matumizi ya L1 au L2. Mofosintaksia ni uhusiano wa kiisimu wa jinsi vipashio vya mofolojia na sintaksia vinavyoungana kuunda sentensi. Nomino ndicho kipashio kikuu cha kimofosintaksia ambacho hutawala maneno mengine katika tungo. Kipashio hiki hutumika kimofosintaksia kudhihirisha uhusiano wa neno kuu na maneno mengine kupitia kwa ngeli ambapo nomino hutawala muundo wa sentensi kwa njia ya upatanishi wa kisarufi.

Katika sarufi ya Kiswahili na sarufi ya lugha zingine za Kibantu, uanianishaji wa nomino ni muhimu sana. Hii ni kwa sababu nomino ndiyo uti wa mgongo wa sarufi ya lugha za Kibantu. Matumizi ya maneno mengine katika tungo kama vile vitenzi, vivumishi na viwakilishi hutegemea ngeli za nomino katika sentensi kuonyesha uhusiano wa kisarufi sanifu (Kihore na wenzake, 2004; Habwe na Karanja, 2004). Nomino zote katika lugha za Kibantu zimeainishwa katika vikundi vya ngeli. Ngeli ni majina ambayo hupatikana katika kundi moja yaliyo na upatanishi wa kisarufi na viambishi ngeli vya idadi ya umoja na wingi unaofanana (Kapinga, 1983; Massamba, 2004; Mgullu, 1999; Okumu, 2006).

Mpangilio wa ngeli hutegemea mfumo fulani ambao ndio msingi wa lugha husika. Luganda na Kiswahili sanifu hupanga na kutumia ngeli zake kwa kuzingatia mfumo wa kimofolojia, kisintaksia na kisemantiki. Matumizi ya ngeli nomino huweza kutaja mofu idadi ya nomino kwa kutumia mofu awali kimofolojia. Mofu hizi za kiidadi kimofolojia hupachikwa kwenye mizizi au mashina ya maneno mengine katika tungo kuwakilisha idadi na vilevile semantiki ya nomino inayozungumziwa katika tungo. Kwa hiyo, ikiwa unatumia nomino au kutaja nomino ni lazima kuitaja kama idadi ya umoja au wingi kimofolojia na kisintaksia kurejelea mantiki na maana ya utungo. Matumizi haya humsaidia mtumiaji kutambua mofolojia ya nomino na upatanishi wake wa kisarufi hasa kwa nomino

11 This work is licensed under a Creative Commons Attribution 4.0 International License. 
ambayo mofu zake za umoja hazidhihiriki kimofolojia bali katika sintaksia na semantiki.

Ngeli za Luganda na Kiswahili sanifu zimeainishwa kwa kuzingatia utaratibu wa kimofolojia wa kupanga ngeli za nomino kuzingatia viambishi awali vya nomino kudhihirisha idadi ya umoja na wingi (Mgullu, 1999; Mathooko, 2004). Utaratibu wa pili wa kisintaksia wa kupanga ngeli nomino hutumia viwakilishi nomino ambavyo huonyesha uhusiano wa kisarufi wa nomino na maneno mengine katika sentensi. Katika mpangilio huu neno kuu ambalo ni nomino hutawala maneno mengine katika sentensi (Kapinga, 1983; Kithuku, 2012).

Viambishi awali vya msingi vya nomino kimofolojia na kisintaksia hutumika kuwakilisha idadi ya umoja na wingi pamoja na kudhihirisha uhusiano wa nomino kutawala maneno mengine katika tungo. Viambishi viwakilishi vya msingi vya nomino vinavyotumika kuonyesha uhusiano wa nomino na maneno mengine katika tungo baina ya lugha hizi huwiana na kutofautiana. Uwiano na tofauti za kanuni za matumizi ya viambishi viwakilishi vya msingi vya nomino ngeli baina ya Luganda na Kiswahili sanifu kimofosintaksia huweza kusababisha mwingiliano na athari katika matumizi ya Kiswahili sanifu. Hii ni kwa sababu hizi ni lugha zinazobainika kuwa lugha mbili tofauti kutokana na sifa za kiisimu zinazopambanua na kuitofautisha kila lugha hizi za Kibantu zenye nasaba moja. Athari za kimofosintaksia za wiano na tofauti za ngeli za Luganda na Kiswahili sanifu unaotokana na kanuni/mifumo za uainishaji na matumizi ya ngeli kimofosintaksia baina ya Luganda na Kiswahili sanifu hazijulikani. Kwa hivyo, makala haya yanatathmini kanuni za kimofosintaksia za Luganda na jinsi zinavyoingiliana na kuathiri matumizi ya Kiswahili sanifu.

Kwa hivyo, makala hii inaweka wazi athari za kimofosintaksia za ngeli za Luganda katika matumizi ya Kiswahili sanifu unaojitokeza katika maandishi na mazungumzo ya wanafunzi wa shule za upili wilayani Kampala, Uganda. Makala haya yanatokana na lengo la kutatmini kanuni za kimofosintaksia za Luganda na jinsi zinavyoingiliana na kuathiri matumizi ya Kiswahili sanifu.

\section{MAPITIO YA MAANDISHI}

Tafiti mbalimbali zimefanyika kudhihirisha athari za L1 katika L2. Niyomugabo (2016) akitathmini makosa na matatizo katika ufundishaji wa Kiswahili katika shule za Rwanda, aligundua kuwa asilimia $78 \%$ ya wanafunzi wanaojifunza Kiswahili walishindwa kumudu mazoezi ya matumizi ya ngeli. Utafiti wake ulidhihirisha athari za ngeli za Kinyarwanda katika Kiswahili sanifu ambapo wanafunzi walishindwa kuanishia na kutambua viambishi viwakilishi vya nomino za wanyama, wadudu na ndege ambayo katika Kiswahili huainishwa katika ngeli ya [a-wa] lakini katika Kinyarwanda yameainishwa katika ngeli tofauti ya Kinyarwanda.

Ntawiyanga (2015) akichunguza mofosintaksia ya ngeli za nomino katika Kiswahili na Kinyarwanda, alibainisha urejeshi wa ngeli unaopatikana katika vitenzi, vivumishi na viwakilishi na jinsi unavyotumika kuonyesha uhusiano wa ngeli nomino kutawala maneno mengine katika sentensi kimofosintaksia. Katika ulinganishi wa mofosintaksia ya lugha hizi mbili, aligundua kuwa, Kiswahili na Kinyarwanda ni lugha za Kibantu ambazo zinatumia mifumo ya ngeli inayofanana sana hasa ya ngeli kutumika kudhihirisha upatanishi wa kisarufi wa nomino na maneno mengine katika tungo. Utafiti wake ulidhihirisha athari za kiisimu za wanafunzi kuhamisha baadhi ya vipengele vya kiisimu vya Kinyarwanda katika matumizi ya Kiswahili, kuchanganya ndimi, kujumuisha kanuni za kisarufi za Kinyarwanda na Kiswahili, kutohoa maneno na kutafsiri miundo ya Kinyarwanda hadi Kiswahili sanifu. Miongoni athari za kiisimu uliojitokeza ni pamoja na uhamishaji wa viambishi vya ngeli ya mahali [pa, ku, mu], ngeli ya vitenzi jina ya $\{\mathrm{ku}\}$, ngeli ya viumbe hai ya [a-wa] na majina ya mkopo yasiyoambishwa viambishi vya idadi vya Kinyarwanda katika matumizi ya Kiswahili sanifu pamoja na kujumlisha viambishi ngeli na kutumia viambishi ngeli vya Kiswahili visivyo mwafaka. Athari hizi zilisababisha miundo tofauti ya matumizi ya ngeli nomino na kuharibu uhusiano wa nomino kuu na maneno mengine katika tungo. Mwanaisimu Adolph (2014), naye akichunguza utohozi wa nomino za Kiswahili na athari yake katika ngeli za kihaya, alibaini kuwepo kwa athari ya ngeli inayotokana na utohozi wa nomino za Kiswahili katika lugha ya Kihaya ambao

12 This work is licensed under a Creative Commons Attribution 4.0 International License. 
uliathiri na kubadili ngeli nomino asili za Kihaya. Utafiti wake ulidhihirisha athari za L2 (Kiswahili) katika L1 (Kihaya) huku makala haya yakitathmini athari za kanuni za matumizi ya kipashio cha ngeli za Luganda katika matumizi ya Kiswahili sanifu.

Akichunguza athari za uhamishaji wa sintaksia ya Kiluo kwa upatanishi wa sarufi ya Kiswahili, Carolyne, Simala na Kobia (2017) waligundua athari za uhamishaji wa sarufi ya Kiluo kwenye sarufi ya Kiswahili ambao ulijitokeza katika viwango vya kisarufi vya ngeli, nafsi na idadi. Katika uchunguzi wake, athari hii iliathiri upatanishi wa kisarufi katika kiwango cha ngeli, ambapo viambishi vya upatanishi vilivyotumiwa katika kundi nomino vilikuwa tofauti na ngeli za nomino za Kiswahili sanifu. Aidha, anaeleza sababu kuwa athari za upatanishi wa Kisarufi wa Kiluo katika Kiswahili ulitokana na tofauti za kanuni za uambishaji za lugha hizi mbili. Mramba (2015), katika uchunguzi wa athari za Kirombo katika ujifunzaji wa Kiswahili alibaini athari zinazotokana na utohoaji wa msamiati, na utofauti wa maumbo ya umoja na wingi ya ngeli kati ya Kirombo na Kiswahili. Kulingana na utafiti wake athari hizi zilipelekea kutokea kwa athari za L1 za kimatamshi, kitahajia, kisarufi na kimsamiati kujitokeza katika ujifunzaji wa Kiswahili sanifu.

Otiende (2013) akichunguza athari za kimofofonolojia za Kiolusuba katika matumizi ya Kiswahili sanifu, alitambua kuwa uainishaji wa majina katika ngeli ndiyo sifa kuu inayozitambulisha lugha za Kibantu. Kutokana na ugunduzi wake, alilinganisha ngeli za Kiolusuba na Kiswahili sanifu na kubainisha ngeli nomino za Kiswahili sanifu na Kiolusuba kwa kuonyesha viambishi vinavyotumika katika uainishaji wa ngeli pamoja na mifano ya nomino zinazopatikana katika kila ngeli hizi kimofolojia. Uchunguzi wake ulibainisha athari za mifanyiko ya kifonolojia baina ya viambishi vya kimofolojia vya ngeli za Kiswahili sanifu na Kiolusuba ambazo zilijitokeza katika imla, mazungumzo na maandishi ya wanafunzi wa shule za msingi ambao L1 ni Kiolusuba. Utafiti wake uligundua kuwa tofauti kati ya mifumo ya fonimu na mifanyiko ya kimofonolojia ya Kiswahili na Kiolusuba, huathiri matumizi ya Kiswahili miongoni mwa wazungumzaji wa Kiolusuba katika vipashio vya kiisimu, ngeli akijidhihirisha kama mojawapo wa kipashio cha kiisimu.
Ame (2017), akichunguza athari za kiisimu za Lahaja ya Kitumbatu katika Kiswahili sanifu, aligundua kuwa nomino nyingi za kitumbatu ni za kawaida na hazitofautiani sana na nomino za Kiswahili sanifu isipokuwa baadhi ya nomino za Kitumbatu huwa katika maumbo ya kilahaja za Kitumbatu na hatimaye kuyafanya makundi ya ngeli za majina kubadilika kwa kuzingatia kigezo cha upatanishi wa kisarufi. Muundo wa ngeli za Kitumbatu huathiri watumiaji wa Kiswahili sanifu wa shule Tumbatu kwa kiasi fulani kwani huwafanya wanafunzi wengi kuingiza mfumo wa lahaja wakati wa kutunga sentensi za Kiswahili sanifu na hivyo kuathiri matumizi ya viambishi vipatanishi vya msingi vya ngeli za Kiswahili sanifu. Barno (2013) Katika uchunguzi wake juu ya muundo wa mofosintakisa wa kitenzi cha Kinandi alitambua kuwa vipengele vya hali, wakati, ukanushi, uyakinishi na vipatanishi vikipachikwa kwenye kitenzi vina uwezo wa kuathiri muundo wa kirai au sentensi katika kiwango cha uamilifu na kuathiri mofosintaksia ya kategoria za kisarufi katika virai na sentensi.

Mukuria (1987) akilinganisha mpangilio wa ngeli za Kikikuyu cha Kiambu na Kiswahili sanifu kisintaksia na kisemantiki kuzingatia vigezo vya idadi za ngeli, wiano wa kisarufi, na misingi ya kimaana inayotokea katika ngeli za lugha hizo. Alitambua kwamba kimuundo kiambishi kilichoko kwenye jina hukaririwa kwenye vipande vingine vya sarufi ambavyo hutawaliwa na jina. Aidha, kisemantiki, kila mojawapo ya ngeli inahusiana na dhana moja au zaidi. Utafiti wake pia ulitambua kuwa lugha za Kibantu kama vile Kiswahili na Kikikuyu zina takiriri za viambishi kwenye vipande vya sarufi. Utafiti wake ulikuwa msingi wa uainishaji wa ngeli za Luganda na Kiswahili Kimofolojia na Kisintaksia kwa ajili ya kutambua athari za lugha mama katika matumizi ya Kiswahili sanifu.

Nyanda (2015) alitambua kuwa mwingiliano baina ya lugha mbili tofauti husababisha kuathiriana kwa lugha kutokana na lugha kuchukua baadhi ya msamiati kutoka lugha nyingine na kuuingiza katika lugha yake. Aidha, anasisitiza kuwa lugha huathiriana katika viwango vya kiisimu kuanzia fonolojia, mofolojia, sintaksia na semantiki kutokana na mwingiliano baina ya wanajamii wa lugha hizo. Vile vile anaeleza kuwa mwingiliano

13 | This work is licensed under a Creative Commons Attribution 4.0 International License. 
baina ya lugha mbili tofauti hutokana na ukaribu ama mwingiliano baina ya wanajamii na lugha. Makutano haya ama ukaribu huu husababisha mwingiliano baina ya lugha husika. Kwa mtazamo wake, wazungumzaji wa lugha zilizo karibu wazungumzapo huwa wanadhihirisha tofauti za kiisimu katika viwango vya kiisimu vya fonolojia, mofolojia, sintaksia na semantiki.

Okumu (2006) katika kuthamini mpangilio wa ngeli kuzingatia mifumo ya uanishaji wa kimofolojia na kisemantiki pamoja na kisintaksia, aligundua kuwa wanafunzi wangali wanatatizwa na funzo la kipashio cha ngeli kimatumizi hasa katika kuendeleza sentensi sahihi kisarufi kutokana na kukanganywa kwao na maumbo yanayotambulisha ngeli pamoja na kutoelewa maana ya ngeli. Alipendekeza haja ya walimu wa Kiswahili kuzua mbinu za kuwawezesha wanafunzi kuelewa maumbo na maana ya nomino hizi na kufanywa utafiti zaidi kuhusiana na ufundishaji wa kipashio cha kisarufi cha ngeli kuimarisha ujifunzaji na kuondoka na na athari za ujumlishaji na uhamishaji wa lugha za kijamii katika matumizi ya Kiswahili sanifu. Pendekezo lililopelekea uchunguzi huu kufanywa kwa ajili ya kuweka wazi athari za lugha mama katika Kiswahili sanifu kuwawezesha wanafunzi na walimu kukuza matumizi bora ya ngeli za Kiswahili sanifu.

Mapitio haya ya maandishi yamebainisha kuwa ingawa kuna tafiti ambazo zimefanyika kuhusiana na uainishaji wa ngeli za Kibantu na athari zake katika matumizi, athari za kimofosintaksia zinazotokana na wiano na tofauti za ngeli za Luganda na Kiswahili sanifu haujawekwa bayana. Kwa hiyo, makala hii inaweka wazi athari za kimofosintaksia za ngeli za Luganda katika matumizi ya Kiswahili sanifu ili kuwawezesha walimu na wanafunzi kuweka mikakati ya kukabiliana nazo na kujenga matumizi bora ya Kiswahili sanifu.

\section{Nadharia za Utafiti}

Utafiti huu uliongozwa na mihimili miwili ya nadharia ya Umilikifu na Unganifu ya Chomsky (1981) kama ilivyoendelezwa na (Shabani, 2015). Mhimili wa kwanza unazingatia sifa za sarufi bia ambao huchukulia kuwa kanuni zilezile za kisintaksia hutumika katika lugha zote, ingawa zinaweza kutofautiana kidogo katika lugha mbalimbali kwa msingi wa data ya lugha hizo. Mhimili wa pili ni wa upatanifu unaofafanua uhusiano wa kimiundo kwa kubainisha sheria ambazo huunganisha vipashio vya sehemu ya sentensi na miktadha ambamo uhusiano huo wa kiunganifu hutumika. Utafiti huu ulishughulikia uhusiano wa kisintaksia ambapo neno moja hutawala umbo la kimofolojia la neno au maneno mengine katika tungo na uwakilishi ngeli kuchukua nafasi ya nomino katika sentensi kuathiri upatanisho wa kisarufi wa maumbo ya maneno mengine katika maandishi na mazungumzo ya wanafunzi (kirejeo sabaki kufungamana na kipashio kinachomilikiwa katika tungo). Uchaguzi wa nadharia hii ulitokana na madai kuwa maarifa ya kisintaksia ya mtu yanaweza kuonyeshwa kupitia njia mbili rasmi za kanuni za kimsingi ambazo hupatikana katika lugha zote (ubia wa kanuni za kisarufi) na tofauti za kimofosintaksia za kanuni miongoni mwa lugha huweza kusababisha mwingiliano wa lugha. Mihimili hii iliusaidia utafiti huu kutambua sifa za kimofolojia na kisintaksia za ngeli za Luganda na Kiswahili na athari za kimofosintaksia za ngeli za Luganda katika matumizi ya Kiswahili sanifu

\section{Mbinu za Utafiti}

Data za utafiti zilikusanywa kutoka maktabani na nyanjani. Data za maktabani zilihusisha kusoma na kuchanganua vitabu na maandishi ya kiisimu yaliyoandikwa kuhusu ngeli za Luganda na Kiswahili sanifu, mijadala na usaili wa walimu wa Kiswahili 30 kutoka shule za upili 15 teule, wilayani Kampala, Uganda. Mijarabu ya kisarufi, mijadala na maandishi ya insha kutoka kwa wanafunzi 117 wa kidato cha tano na sita ulitumika kuchanganua athari za ngeli za Luganda katika Kiswahili sanifu. Idadi lengwa ilikuwa shule za upili 46, walimu 160 na wanafunzi 515. Mbinu ya kimakusudi ilitumika kuteua: Shule 15 ambazo zinafundisha Kiswahili katika kidato cha tano na sita; walimu 2 kutoka kila shule lengwa ambao L1 ni Luganda na walimu wa Kiswahili sanifu, na wanafunzi wote wa kidato cha tano na sita ambao L1 ni Luganda na watumiaji wa Kiswahili kama L2 walihusika katika utafiti huu. Muundo wa kimaelezo ulitumika kutathmini kanuni na kueleza athari za ngeli za Luganda katika matumizi ya Kiswahili sanifu. Muundo huu uliteuliwa kutoa picha halisi ya jinsi wanafunzi wanavyotumia ngeli katika sentensi za Kiswahili

14 | This work is licensed under a Creative Commons Attribution 4.0 International License. 
sanifu na vilevile kutoa msingi wa sifa za matumizi ya ngeli kama zinavyotumika na wanafunzi na kuelezwa na walimu (Momamnyi, Ambuyo na Chimera 2019; Mugenda 2008; na Hendrick, Brickman na Debra 1993). Data iliyoelezwa ilihusu athari za ngeli za Luganda katika matumizi ya Kiswahili sanifu kutokana na uhamishaji wa kanuni na maumbo ya kimofosintaksia ya ngeli za Luganda katika matumizi ya Kiswahili sanifu. . Data hii ilipatikana baada ya kuthmini kanuni za matumizi ya ngeli za Luganda na Kiswahili sanifu.

Uchanganuzi wa data ulianza kwa kuainisha ngeli za Luganda na Kiswahili kimofosintaksia, kutambua uwiano wa maumbo ya ngeli za Luganda na Kiswahili kimofosintaksia, kutathmini kanuni za kimofosintaksia zinazoingiliana na kuathiri matumizi ya ngeli za Luganda katika Kiswahili na kubainisha athari husika kama zinavyotumika na wanafunzi katika maandishi na mijadala yao katika vikundi na darasani. Athari za ngeli za Luganda katika matumizi ya Kiswahili sanifu yalitathminiwa kwa kuzingatia mihimili ya ubia na upatanisho ya nadharia ya Umilikigu na unganifu ya Chomsky (1981).

Mfano (1):

Mwingiliano wa Luganda kwa Kiswahili

a) $\mathrm{Ng}$ 'ombe zimeletwa nyumbani [n-zi]

b) Mbuzi zinakula majani [n-zi]

c) Paka zimekamata panya [n-zi]

Tofauti baina ya ngeli za Luganda na Kiswahili sanifu ilibainika katika kiwango cha uambishaji nomino na maneno mengine kama vitenzi, vivumishi na viwakilishi kuwakilisha dhima ya kisarufi ya idadi ya umoja na wingi. Uchanganuzi wa ngeli baina ya Luganda na Kiswahili sanifu

Mfano (2):

Mwingiliano wa Luganda kwa Kiswahili

a) Mukate gumeisha jokoni [mu-gu]

b) Miti gimekatwa [mi-gi]

c) Mugulu gunaluma [mu-gu]

d) Maguu gananiuma [ma-ga]

Kudhihirisha Athari katika Matumizi kimazungumzo na Kimaandishi. Data za kimazungumzo zilitokana na mijadala ya wanafunzi darasani na maandishi ilitokana na mojarabu ya kisarufi kuhusu ngeli na maandishi ya insha. Athari
Matokeo ya Athari za Kimofosintaksia za Ngeli za Luganda katika Matumizi ya Kiswahili Sanifu

Matokeo ya utafiti yalidhihirisha athari za kimofosintaksia za ngeli za Luganda katika matumizi ya Kiswahili sanifu. Athari hizi zilitokana na uwiano na tofauti za ngeli za Luganda na Kiswahili sanifu. Utafiti huu ulitambua kuwa Luganda na Kiswahili hutumia mfumo wa kimofolojia na kisintaksia katika uanishaji na matumizi ya ngeli nomino. Kwa kuongozwa na mhimili wa kwanza wa nadharia ya umilikifu na ufungami (Chomsky, 1981) unaozingatia sifa za sarufi bia na ambao huchukulia kuwa kanuni zilezile za kimofosintaksia hutumika katika lugha zote, ingawa zinaweza kutofautiana kidogo katika lugha mbalimbali kwa msingi wa data ya lugha hizo. Utafiti huu ulibainisha kuwa, nyingi kati ya ngeli za Luganda na Kiswahili sanifu zina sifa nyingi zinazofanana kimofolojia na kisintaksia ingawa zinatofautiana sana katika kiwango cha viambishi vipatanishi vya msingi vya nomino pamoja na maana. Kama ilivyodhihirika katika mifano ya sentensi za wanafunzi katika ngeli ya [nzi ya Luganda na [a-wa] ya Kiswahili.

Maumbo sanifu ya Kiswahili sanifu

Ng'ombe wameletwa nyumbani [Ø-wa]

Mbuzi wanakula majani. [Ø-wa]

Paka wamewakamata panya [Ø-wa]

uligundua kuwa, licha ya kuwa Luganda na Kiswahili ni lugha za nasaba moja za Kibantu, ulinganishi na ulinganua wa ngeli za Luganda na Kiswahili sanifu kimofolojia na kisintaksia ulidhihirisha uwiano na tofauti ambazo ziliathiri matumizi ya Kiswahili sanifu. Kama katika

Maumbo sanifu ya Kiswahili sanifu

$\begin{array}{ll}\text { Mkate umekwisha jikoni } & {[\mathrm{m}-\mathrm{u}]} \\ \text { Miti imekatwa } & {[\mathrm{mi}-\mathrm{i}]} \\ \text { Mguu unauma/ unaniuma } & {[\mathrm{m}-\mathrm{u}]} \\ \text { Miguu inaniuma } & {[\mathrm{mi}-\mathrm{i}]}\end{array}$

za uwiano na tofauti za ngeli za Luganda katika maandishi na mazungumzo ya Kiswahili sanifu yalijitokeza kutokana na mifumo ya uainishaji na uambishaji wa viambishi vipatanishi vya msingi vya nomino kuu katika maneno mengine ya kisarufi ya

15 This work is licensed under a Creative Commons Attribution 4.0 International License. 
Kiswahili katika tungo. Athari hizi zilitokana na kuhamisha viambishi vipatanishi vya nomino za Luganda katika matumizi ya Kiswahili sanifu visivyo sahihi katika tungo kuonyesha uhusiano wa neno kuu na maneno mengine katika tungo.

Wanaisimu linganisha wa lugha za Kibantu huelezea kuwa tofauti za maumbo na miundo ya lugha asili huathiri lugha ya Kiswahili kutokana na tofauti za mifumo (Carolyne na wenzake, 2017). Hata hivyo, katika utafiti huu, ilibainika kuwa sio tofauti tu za mifumo ya uainishaji wa nomino bali hata uwiano wa mifumo ya uainishaji na uambishaji wa viambishi vya ngeli nomino za Luganda na Kiswahili sanifu ulisababisha athari za kimofosintaksia za ngeli nomino katika viwango anuwai. Uhamishaji huu wa viambishi ngeli vya msingi vya nomino za Luganda hadi kwenye lugha ya Kiswahili sanifu kudhihirisha sifa za idadi na jinsia ya kisarufi ya umoja na wingi wa nomino na maneno mengine katika tungo uliathiri matumizi ya ngeli nomino za Kiswahili sanifu katika tungo na kusababisha matumizi yasiyo sanifu. Athari za uwiano na tofauti za ngeli nomino za Luganda na Kiswahili sanifu ilidhihirika katika mijarabu ya Mfano: (3)

a) Mbuzi emepelekwa malishoni kula nyasi

b) Mbuzi imepelekwa malishoni kula nyasi

c) Mbuzi zimepelekwa malishoni kula nyasi

Mwingiliano wa maumbo ya kimofolojia na kisintaksia ya Luganda na Kiswahili ulisababisha uhamishaji maumbo ya ngeli za Luganda kimofosintaksia katika matumizi ya Kiswahili sanifu ambapo maumbo yanayopachikwa kwenye mzizi wa nomino kuwakilisha idadi ya umoja na wingi yanatofautiana hata ingawa watumiaji huyatazama kama alomofu ya mofimu moja. Kwa mfano, maumbo ya ngeli ya tatu umoja [mu] ya Luganda na $[\mathrm{m}]$ ya Kiswahili sanifu, ngeli ya kimofolojia ya nomino ya nne [mi] ya Luganda na [mi] ya wingi, ngeli ya tano [li] ya umoja katika Luganda na [li] ya wingi katika Kiswahili sanifu,

Mfano (4):

(4a)

Mwingiliano

Mutotomuzuri

Muwalimumurefu

Mupolisi/wapolisi

Mudaktari/wadaktari kisarufi, maandishi ya insha na mazungumzo ya wanafunzi ni:

Athari za Uhamishaji Maumbo ya Ngeli Nomino za Luganda katika Kiswahili Sanifu

Athari za maumbo ya viambishi vya Luganda ulijitokeza katika matumizi ya Kiswahili sanifu na hivyo kuathiri usanifu wa matumizi ya Kiswahili sanifu. Athari hii ilitokana na kuwa maumbo na taratibu za mpangilio wa ngeli za Luganda na Kiswahili sanifu unaotumiwa kuonyesha uhusiano wa nomino kuu na maneno mengine katika tungo hutofautiana hata ingawa baadhi ya maumbo yanayotumika yanafanana. Utafiti huu ulitambua kuwa kuna maumbo ya ngeli za Luganda na Kiswahili ambayo hufanana ingawa yanatumia nomino za aina tofauti kisemantiki. Maumbo haya husababisha mwingiliano wa kimofosintaksia unaojitokeza katika matumizi baina ya Luganda na Kiswahili sanifu katika tungo. Mfano ulijitokeza katika ngeli ya i-zi na ngeli ya a-wa ya Kiswahili, ngeli ya li- ya ya Luganda na Kiswahili. kama inavyojitokeza katika mifano katika mfano.

Mbuzi amepelekwa malishoni [en-a] Mbuzi amepelekwa malishoni [i-a] Mbuzi wamepelekwa malishoni [zi-wa]

ngeli ya kimofolojia ya sita ya umoja [ma] ya Luganda na Kiswahili sanifu, ngeli ya saba ya nomino umoja [ki] ya Luganda na Kiswahili sanifu, ngeli ya tisa ya nomino nazali za Luganda [n] umoja na [zi] ya upatanisho na ngeli ya $\mathrm{n} / \varnothing$ ya umoja na [zi] ya wingi, ngeli ya kumi ya wanyama ya [n na zi] ya umoja na wingi ya Luganda na ngeli ya [n/ Ø] ya umoja na [zi] ya wingi isiyo ya wanyama na mwisho ngeli ya [ku na mu] ya kitenzi jina na mahali ndani. Maumbo haya yanafanana ila yanatumia nomino ambazo zinatofautiana kisemantiki kwa mjibu wa Luganda na Kiswahili sanifu kama inavyodhihirika katika

16 This work is licensed under a Creative Commons Attribution 4.0 International License. 
(4b)

Muti mukubwa

Mukati/mugati mutamu

Muguugubwa

(4c)

Vitabu byangu

Kiatu yangu

Viatu zangu

(4d)

Maji mangi/maingi

Mayai mangi/maingi

Mayai mengi

Sikio kupana Sikio pana (semantiki [ku] ya sikio

(4e)

Mwingiliano wa Luganda kwa Kiswahili

d) Ng'ombe zimeletwa nyumbani [n-zi]

e) Mbuzi zinakula majani [n-zi]

f) Paka zimekamata panya [n-zi]

(4f)

Mwingiliano wa Luganda kwa Kiswahili

a) Macho gananiuma/ganauma [ma-ga] [li-ma] [li-ya]

[li-ga]

(4g)

Mwingiliano wa Luganda kwa Kiswahili

e) Mukategumeisha jokoni [mu-gu]

f) Miti gimekatwa [mi-gi]

g) Mugulu gunaluma [mu-gu]

h) Maguu gananiuma [ma-ga]

Matumizi haya yalidhihirisha kuwa nomino za Luganda na Kiswahili sanifu hutumia viambishi awali vya ngeli kuainisha nomino kimofolojia na kisintaksia. Miundo hii ya matumizi ya viambishi hutofautiana. Tofauti zinazojitokeza ni za kimaumbo ambazo hutumika kuwakilisha ngeli na idadi ya umoja na wingi wa nomino. Viambishi vya Luganda vilivyotumika katika tungo kuwakilisha ngeli na idadi ya umoja na wingi kutokana na neno kuu viliathiri mofosintaksia ya Kiswahili sanifu kutokana na matumizi ya maumbo wakilishi ya ngeli nomino yaliyokuwa na muundo tofauti ingawa yalikaribiana kufanana au kufanana kimatamshi au kifonimu.

Athari ya Ujumlishaji wa Maumbo ya Viambishi Awali vya Ngeli vya Luganda katika Kiswahili Sanifu

$$
\begin{array}{ll}
\text { m-mi } & \text { [mu-mi ya Luganda] } \\
\text { m-mi } & {[\text { mu-mi ya Luganda] }} \\
& \text { [gu-ga ya Luganda] }
\end{array}
$$

$$
\begin{array}{ll}
\text { ki-vi } & \text { [ki-bi ya Luganda] } \\
\text { Ki-vi } & \text { [ki-bi ya Luganda] } \\
\text { ki-vi } & \text { [ki-bi ya Luganda] }
\end{array}
$$

$$
\begin{aligned}
\text { ji-ma } & \text { [ma ya Luganda] } \\
\text { ji-ma } & \text { [ma ya Luganda] } \\
\text { [okutu ku...] } & \text { [ku-ma ya Luganda }
\end{aligned}
$$

Maumbo sanifu ya Kiswahili sanifu

Ng'ombe wameletwa nyumbani

[Ø-wa]

Mbuzi wanakula majani. [Ø-wa]

Paka wamewakamata panya [Ø-wa]

Maumbo sanifu ya Kiswahili sanifu

Macha yananiuma /yanauma

$[\varnothing / \mathrm{ji}-\mathrm{ma}]$ $\begin{array}{lr}\text { Maumbo sanifu ya Kiswahili sanifu } \\ \text { Mkate umekwisha jikoni } & {[\mathrm{m}-\mathrm{u}]} \\ \text { Miti imekatwa } & {[\mathrm{mi}-\mathrm{i}]} \\ \text { Mguu unauma/ unaniuma } & {[\mathrm{m}-\mathrm{u}]} \\ \text { Miguu inaniuma } & {[\mathrm{mi}-\mathrm{i}]}\end{array}$

Wanafunzi walidhihirisha ujumlishaji maumbo ya viambishi awali vya ngeli za maneno kwa kuchanganya maumbo awali ya viambishi awali vya Luganda katika Kiswahili sanifu. Uchanganyaji huu wa viambishi awali msingi wa maumbo ya viambishi vya ngeli za Luganda na Kiswahili katika maneno na tungo za Kiswahili sanifu hukuonyesha mahusiano ya karibu ya nomino kuu na maneno mengine katika tungo. Uchanganyaji huu uliathiri matumizi ya Kiswahili sanifu. Ujumlishaji na uchanganayaji wa viambishi awali vya ngeli ulijitokeza katika ngeli hasa zilizo na ukuruba wa kimaumbo kama vile ngeli za [a-wa], [m-mi], [kivi], [ø /ji-ma] kama inavyojitokeza katika mifano kama katika mfano Na.(5a-5e):

17 This work is licensed under a Creative Commons Attribution 4.0 International License. 
Mfano (5):

Mwingiliano wa Luganda kwa Kiswahili

$\begin{array}{lll}\text { a) } & \text { Wamikategimeletwa } & \text { [wa-gi] } \\ \text { b) } & \text { Wamutotobanayimba } & {[\mathrm{wa}-\mathrm{ba}]} \\ \text { c) } & \text { Kiongozi kinaongeya } & {[\mathrm{ki}-\mathrm{ki}]} \\ \text { d) } & \text { Kifaru kinakimbia } & {[\mathrm{ki}-\mathrm{ki}]} \\ \text { e) } & \text { Macho gananiuma } & {[\text { ma-ga }}\end{array}$

Wanafunzi walitumia kiambishi cha wingi [wa] katika kishazi cha (5a na5b) kwa sababu ya maumbo ya ngeli ya kwanza ambayo wingi wa [n] ni [wa] kwa hiyo badala ya wanafunzi hawa kuitumia vyema walijumlisha tu kanuni hii na kuambisha nyuma ya viambishi vyovyote vinavyoanza na umbo [m]. Aidha, badala ya kutumia viambishi vya upatanishi wa kisarufi unaotokana na nomino kuu ya Kiswahili vya [mi] ya kimofolojia leksia na [i] ya upatanishi wa kisarufi katika (5a) na umbo [wa] la kimofolojia leksia kuwakilisha umoja na umbo [wa] ya upatanishi wa nomino kisintaksia, walitumia viambishi vya upatanishi vya wingi kutoka L1 [wagi] na [wa-ba] ambayo ni Luganda sanifu.

Katika mifano ya (5c-5d), wanafunzi walihamisha kanuni ya Kiswahili kuwa kila nomino inayoanza na kiambishi [ki] hubadilishwa na kuwa [vi] katika wingi. Hali hii ni ya ujumlishaji kwa sababu haikuzingatika maumbo ya majina au nomino ya viumbe hai ambao hutumia kiambishi [ki-vi] kwa umoja na katika upatanishi wa kisarufi kutumia viambishi vya ngeli ya 1 na 2 [a-wa]. Maumbo haya ni viwakilishi vya wingi katika Luganda hata hivyo sivyo vya Kiswahili na haionyeshi uhusiano wa nomino kuu ya Kiswahili na vitenzi ambapo vimeambishwa. Mfano wa (5e) unaonyesha matumizi ya viambishi katika maneno kwa sababu ya mfanano wa maumbo ya maneno katika umoja

Mfano (6):

Matamshi na maandishi

a) Watoto $\mathrm{w}$-[a-i]-ngi walikuja darasani. Watoto $\mathrm{w}$-[e]-ngi walikuja darasani.

b) Mayai m-[a-i]-ngi yameoza.

Ubadilishanaji sauti badala ya [wa]

c) Wapolisiwalibakamataw[-a-i]-zi.

d) Leta vitu [mu]-[bi]-ingize ndani.
Maumbo sanifu ya Kiswahili sanifu

Mikate imeletwa [mi-i]

Wanafunzi wanaimba [wa-wa]

Kiongozi anaongea [ki-wa]

Kifavuanakimbia [ki-a]

Macho yananiuma [ma-ya]

na wingi wa Luganda. Wanafunzi walihamisha kiambishi kipatanishi cha wingi [gi] kwenye kitenzi cha nomino kuu kwa sababu ya umbo la nomino [macho] ambalo linakaribiana na la Luganda ambalo ni [maaso]. Ujumlishaji na kufananisha maumbo ya nomino pamoja na kutumia maumbo ya Kiswahili sanifu visivyo katika tungo za kimofosintaksia yaliathiri matumizi ya Kiswahili sanifu.

Athari za Uhamishaji wa Mofofonolojia ya Luganda katika Matumizi ya Kiswahili Sanifu

Ngeli za Luganda na Kiswahili zimeundwa kwa maumbo ya kimofofonolojia ambayo matumizi yake yasipozingatiwa kimaandishi, sifa za lugha husika huathirika na kupoteza usanifu wake. Utafiti huu ulitambua wanafunzi wa Kiswahili ambao L1 ni Luganda wakihamisha matamshi ya Luganda katika matumizi ya Kiswahili, kubadilisha sauti kwa mujibu wa L1, kushindwa kutambua kanuni za kifonolojia zinazoongoza baadhi ya maneno na kuyatumia jinsi yalivyo katika tungo za kimofosintaksia za Kiswahili sanifu. Hali hii ilipotosha usahihi wa matumizi ya maumbo ya ngeli za Kiswahili sanifu kimofofonolojia kwa sababu sauti zenyewe zilikiukwa kimaandishi na kimatamshi kama katika mfano Na.(6a-6h):

18 | This work is licensed under a Creative Commons Attribution 4.0 International License. 
Kubadilisha matamshi ya viambishi; mofimu [ki-] na kutumia [ch] na [cha] kuandikwa kama [kya], [che] kuandikwa kama [kye]

e) [Kya]-kula chimeivachilete

f) [Chi]-kombe [kye]-usi [chi]-mepasuka

[Cha]-kula kimeiva kilete [ky-ch]

[Ki]-kombe [che]usi [ki]-mepasuka.

Kukiuka kanuni ya kudondosha fonimu [-u] inayofuatwa na konsonanti

g) $[\mathrm{Mu}]$-kate $[\mathrm{mu}]$-zuri. Mkate $[\mathrm{m}]$-zuri.

h) $\quad \mathrm{M}[-\mathrm{u}]$-walimu muzuri. $\quad[\mathrm{Mw}]$-alimu $[\mathrm{m}]-$-zuri.

Kupitia mbinu ya majadiliano ya vikundi, wanafunzi walidhihirisha ukiukaji wa kutumia mofimu ya viambishi vipatanishi vya kimofosintaksia vya luganda katika mazungumzo na maandishi ya kiswahili sanifu. vivumishi vingi vilipokea ukiukaji wa kanuni za kifonolojia kama katika vivumishi vya $[a, b, c$, na f]. katika ubadilishanaji wa sauti, wanafunzi walihamisha mofimu za viambishi vya luganda vinavyokaribiana kimaumbo, kimatamshi na kiuanishaji na kuitumia katika kiswahili sanifu. kwa mfano katika [c-h]. matumizi haya yalionyesha ukiukaji wa matumizi ya maumbo na matamshi sahihi ya viambishi vya ngeli nomino ambavyo vimejengwa kimofofonolojia kuwakilisha idadi ya umoja na wingi wa nomino kuegemea sifa za kiswahili sanifu. ukiukaji huu ulisababisha athari za kimofosintaksia za tahajia na maendelezo yanayoonyesha matumizi mabaya ya kiswahili sanifu.

Athari ya Uhamishaji kwa Upatanishi wa Kisarufi

Mfano (7):
a) Muwalimumuzuriønapendawewe.
b) Mayai mangi/maingigamepasuka.
c) Maji Mangi/maingiimemwagika.
d) Bikapobikubwabyangubinapendeza.
e) Byatubyanguzimeribika.

Mifano hii inadhihirisha ukiukaji wa maumbo ambayo yanafaa kuambisha kwenye nomino na vivumishi kuonyesha uhusiano wa nomino na vivumishi. Maumbo ya nomino yaliyotumika katika [a-e] ni maumbo ya Luganda ambayo hayadhihirishi umiliki na usabaki wa nomino kuu na vivumishi katika tungo. Hii ni kwa sababu maumbo yaliyotumika kwenye nomino na kivumishi hayakuwa maumbo ya kifonolojia ya ngeli ya Kiswahili sanifu. Aidha, wanafunzi walihamisha maumbo ya Luganda katika matumizi ya nomino na vivumishi vya Kiswahili, kushindwa kutambua
Kupitia kwa mijarabu ya kisarufi kwa wanafunzi na majadiliano ya moja kwa moja na walimu kuhusu matumizi ya ngeli kudhihirisha upatanisho wa kisarufi, Utafiti uligundua kwamba uhamishaji wa sarufi ya Luganda kwenye sarufi ya Kiswahili huathiri upatanishi wa kisarufi katika Kiswahili kwa njia tatu: upatanishi kati ya nomino na kivumishi, upatanishi kati ya nomino na kitenzi na upatanisho kati ya kiwakilishi na kisabaki chake. Uchunguzi wa maandishi na mazungumzo ya wanafunzi ilibaini kuwa; wanafunzi walihamisha viambishi vya ngeli nomino vya Luganda katika matumizi ya Kiswahili sanifu kudhihirisha upatanishi wa nomino na vivumishi katika tungo. Hali ilikiuka kanuni za matumizi sahihi ya maumbo ya ngeli nomino kuwakilisha umiliki na usabaki wa neno kuu katika kirai nomino $(\mathrm{KN})$ hupatana kisarufi na vivumishi vyake kuhusu vipengele kama vile ngeli, idadi ya umoja na wingi na nafsi. Matumizi ya maumbo ya ngeli za Luganda yaliyodhihirika na kuathiri matumizi ya Kiswahili sanifu katika maandishi na mazungumzo ni kama katika mfano Na.(7a-7e):

$$
\begin{array}{ll}
\text { Mwalimu mzuri anakupenda. } & {[\mathrm{mu}-\mathrm{m}]} \\
\text { Mayai mengi yamepasuka. } & {[\mathrm{ma}-\mathrm{me}]} \\
\text { Maji mengi yamemwagika. } & {[\mathrm{ma}-\mathrm{me}]} \\
\text { Vikapu vikubwa vyanguvinapendeza. } & {[\mathrm{bi}-\mathrm{v}]} \\
\text { Viatu vyanguvimeharibika. } & {[\mathrm{by}-\mathrm{vi}]}
\end{array}
$$

kanuni za kimofofolojia zinazotokea katika baadhi ya viambishi awali vya nomino [b na c] Kwa mfano walitumia kivumishi [maingi] jinsi kilivyo bila kutambua mabadiliko ya kifonolojia, ambapo [a + i] huunda fonimu mpya /e/ na badala yake kutumia aidha kivumishi jinsi kilivyoambishwa au kutumia maumbo ya Luganda [mangi] katika Kiswahili sanifu.

Kuhusiana na uambishaji wa nomino na vitenzi kudhihirisha umiliki na usabaki, wanafunzi walidhihirisha kutumia sifa za kimofolojia za

19| This work is licensed under a Creative Commons Attribution 4.0 International License. 
nomino za Luganda na kuzilinganisha na Kiswahili sanifu na kuhamisha viambishi ngeli vipatanishi katika matumizi ya nomino husika katika tungo. Kwa mfano, walihamisha kiambishi kipatanishi cha ngeli ya 10 [zi] katika ngeli za Kiswahili zinazotumia kiambishi awali kipatanishi cha [wa]. Vilevile, wanafunzi walihamisha maumbo ya upatanishi ya Luganda katika matumizi ya nomino za Kiswahili kwa umoja na wingi na kukiuka kanuni

Mfano (8):

Mwingiliano wa Luganda kwa Kiswahili

a) $\mathrm{Ng}$ 'ombe zimeletwa nyumbani [n-zi]

b) Paka zimekamata panya [n-zi]

c) Meno gananiuma [ma-ga]

d) Muti gumevunjika [mu-gu]

e) Miti gimeanguka [mi-gi]

Kirai cha [8a na 8b] kilidhihirisha kanuni ya uhamishaji wa maumbo ya kisemantiki ya Luganda katika Kiswahili sanifu. Wanafunzi walifasiri na kubeba viambishi vipatanishi vya ngeli ya 10 [n-zi] ya Luganda katika matumizi ya nomino katika ngeli ya 1 na 2 kisemantiki. Tungo za [c-d] zilidhihirisha wanafunzi kuhamisha maumbo ya kimofolojia na kisintaksia yanayodhihirisha upatanishi wa kisarufi wa nomino zilizoainishwa katika ngeli sawa na Kiswahili sanifu [3 na 4] ya Luganda katika matumizi ya Kiswahili sanifu. Matumizi haya yalikiuka kanuni ya umiliki na usabaki ambapo nomino kuu huathiri maneno mengine katika tungo.

Athari za Kimuundo za Uongezaji Fonimu za Luganda Katika Nomino za Kiswahili Sanifu

Nomino za Kiswahili na za Luganda hutofautiana kimuundo. Uchunguzi wa maandishi na mazungumzo ya wanafunzi yalidhihirisha kuwa nomino za Kiswahili sanifu yanayoanza na kiambishi awali $[\mathrm{m} / \mathrm{mw}$ na $\mathrm{m}]$ hutofautiana na ya ya matumizi ya viambishi vipatanishi vya Kiswahili vinavyokubalika. Athari hii ilijitokeza katika ngeli ya [m-mi/u-i], [ki-vi], [ji-ma/li-ya], [n-zi/i-zi na uzi]. Matumizi haya yaliathiri upatanishi wa Kiswahili sanifu kwa sababu baadhi ya viambishi vya upatanishi wa kisarufi havikuzingatia umantiki wa matumizi na upatanishi wa kisarufi wa tungo katika Kiswahili sanifu kimaandishi kama katika mfano Na.(8a-8e):

$$
\begin{array}{ll}
\text { Maumbo sanifu ya Kiswahili sanifu } \\
\multicolumn{3}{l}{\text { Ng'ombe wameletwa nyumbani }} & {[\varnothing-\text {-wa }]} \\
\begin{array}{ll}
\text { Paka wamewakamata panya } & {[\varnothing-w a]} \\
\text { Meno yananiuma } & {[\text { ma-ya] }} \\
\text { Mti umeanguka } & {[\mathrm{M}-\mathrm{u}]} \\
\text { miti imeanguka } & {[\mathrm{mi}-\mathrm{i}]}
\end{array}
\end{array}
$$

Luganda ambayo huongeza irabu /u/ mbele yake na kuwa [mu/muwa na mu]. Tofauti za muundo huu wa nomino unapotumika katika maandishi hubainisha ikiwa lugha iliyotumika ni Kiswahili au Luganda. Kwa mujibu wa utafiti huu, wanafunzi waliongeza irabu /u/ mbele ya muundo wa nomino zinazoanza na konsonanti $/ \mathrm{m} /$. Walitumia kanuni ya kifonolojia ya uchopekaji au uongezaji wa irabu /u/ kuvunja mkabiliano wa king'ong'o $/ \mathrm{m} /$ na konsonanti halisi za Kiswahili sanifu. Hii ni kwa sababu Luganda hairuhusu mofu $/ \mathrm{m} /$ kufuatana na konsonanti halisi moja kwa moja. Kwa hiyo, wanafunzi ambao L1 ni Luganda walitumia kiambishi [mu] katika nafasi ya [m] ya nomino za Kiswahili sanifu kutokana na kuhamisha muundo wa nomino za Luganda. Matumizi haya yaliathiri ngeli nomino za ngeli ya 1 na 3 ya Kiswahili sanifu. Matumizi ya uongezaji wa irabu /u/ uliathiri umiliki na usabaki wa maneno mengine kama vile vivumishi katika tungo na hivyo kuathiri mofosintaksia ya Kiswahili sanifu. Mifano ya $\mathrm{Na}(9 \mathrm{a}-9 \mathrm{f})$ inadhihirisha athari husika.
Mfano (9):

$\begin{array}{lll}\text { a) } & \text { Mtoto } & \text { Mutoto* } \\ \text { b) } & \text { Mti } & \text { Muti* } \\ \text { c) } & \text { Mlima } & \text { Mulima* } \\ \text { d) } & \text { Mvulana } & \text { Muvulana* } \\ \text { e) } & \text { Mwezi } & \text { Muwezi* } \\ \text { f) } & \text { Mlingoto } & \text { Mulingoti* }\end{array}$

20 This work is licensed under a Creative Commons Attribution 4.0 International License. 
Athari za Uchopekaji wa Viambishi Awali vya Luganda katika Nomino za Kiswahili Sanifu

Kimsingi, maumbo ya kimofolojia ya nomino za Kiswahili sanifu yamegawanywa katika aina kuu mbili; maumbo ya nomino ambayo huambikwa viambishi awali vinavyobeba seti za sifa za kisarufi na maumbo ya nomino yasiyoambikwa viambishi awali vinavyoashiria idadi ya umoja na wingi. Athari ya uchopekaji wa viambishi awali vinavyowakilisha idadi ya umoja na wingi ilijitokeza katika nomino za Kiswahili ambazo haziambishwi mofimu za idadi. Mengi ya maneno haya ni maneno ya mkopo yanayopatikana katika Kiswahili sanifu. Kimofolojia na kisarufi maneno haya huwakilishwa na maumbo kapa. Wanafunzi walibadilisha maumbo ya kimofolojia ya maneno yasiyo na maumbo ya viambishi vya idadi ya umoja na wingi kwa kuambisha maumbo ya Luganda kuwakilisha idadi na katika hali fulani waliyadondosha kimuktadha kutimiza upatanifu wa kisarufi katika Kiswahili sanifu.

Athari hizi zilijitokeza katika nomino za ngeli ya 1 na 2 [M-wa] za maneno ya mkopo hasa ya wafanyabiashara na majina ya wataalamu. Kwa mjibu wa mofolojia ya maneno haya, umbo kapa hutumika kuwakilisha umoja na umbo [ma] kuongezwa nyuma yake kuwakilisha wingi. Hata hivyo, wanafunzi waliongeza kiambishi cha Luganda [mu] kuwakilisha umoja na kutumia kiambishi cha ngeli ya wingi [wa] badala ya kutoambisha kiambishi awali [ma] nyuma ya nomino mkopo ya viumbe wenye uhai kuwakilisha wingi kama inavyokubalika katika Kiswahili sanifu. Matumizi haya yalikiuka matumizi sahihi ya nomino hizi na kuathiri mofosintaksia ya matumizi ya nomino mkopo katika tungo za Kiswahili sanifu kama inavyodhihirishwa katika mfano Na. (10a10e).

Kwa mfano (10):

\section{Mwingiliano}

a) Mupolisi/wapolisi

b) Mudaktari/wadaktari

c) Mufundi/wafundi

d) Museremala/waseremala

e) Muserikali/waserekali
Kiswahili sanifu

Øpolisi/ mapolisi

Ødaktari/ Madaktari

Øfundi/mafundi

Øseremala/maseremala

Øaskari/maaskari $\begin{array}{ll}\text { Ngeli } & \\ \text { m-wa } & \text { [mu-ba ya Luganda] } \\ \text { m-wa } & \text { [mu-ba ya Luganda] } \\ \text { m-wa } & \text { [mu-ba ya Luganda] } \\ \text { m-wa } & \text { [mu-ba ya Luganda] } \\ \text { m-wa } & \text { [mu-ba ya Luganda] }\end{array}$

tofauti na ngeli ya nomino katika kikundi nomino cha Kiswahili sanifu. Vilevile, athari za uhamishaji wa maumbo ya ngeli za Luganda yalidhihirika katika matumizi ya viambishi vinavyokaririwa kwenye vivumishi kuwakilisha umiliki na usabaki wa nomino kuu na maneno mengine katika tungo. Athari za ujumlishaji wa maumbo katika matumizi ya ngeli za Kiswahili, kuchanganya maumbo ya Luganda na Kiswahili katika tungo za Kiswahili, uchopekaji wa mofimu uliojitokeza katika matumizi ya viambishi ngeli vya Luganda viliathiri matumizi sahihi ya tungo za Kiswahili sanifu. Aidha, athari za uwiano na tofauti za ngeli za Luganda katika maandishi na mazungumzo ya Kiswahili sanifu yalijitokeza kutokana na mifumo ya uainishaji na uambishaji wa viambishi vipatanishi vya msingi vya nomino kuu katika maneno mengine ya kisarufi ya Kiswahili katika tungo. Utafiti huu, pia ulibaini kuwa sio tofauti tu za mifumo ya uainishaji wa nomino huathiri matumizi ya vipashio vya kisarufi katika tungo, bali

21 This work is licensed under a Creative Commons Attribution 4.0 International License. 
hata uwiano wa mifumo ya uainishaji na uambishaji wa viambishi vya ngeli nomino za Luganda na Kiswahili sanifu husababisha athari za kimofosintaksia za ngeli nomino katika vipashio vya kisarufi anuwai.

\section{MAPENDEKEZO}

Utafiti huu ulichunguza athari za kimofosintaksia za ngeli za Luganda katika matumizi ya Kiswahili sanifu miongoni mwa wanafunzi shuleni. Kunapendekezwa tafiti nyinginezo kufanyika katika kipashio kingine cha kimofosintaksia kwa ajili ya kubaini athari za vipashio vya kisarufi vya lugha ya Kwanza kwa lugha ya pili au kinyume. Aidha, utafiti huu ulichunguza vipengele vya kimofolojia na kisintaksia, utafiti mwingine unaweza kufanyika katika vipengele vingine vya uainishaji wa ngeli vya semantiki au vyote kubaini athari za Luganda katika Kiswahili sanifu ama lugha nyingineyo ya Kibantu na Kiswahili sanifu kwa madhumuni ya kutambua athari za lugha ya mama katika matumizi ya Kiswahili kwa ajili ya kukuza matumizi sahihi ya Kiswahili sanifu.

\section{MAREJELEO}

Adolph, E. (2014). Utohozi wa nomino za kiswahili na athari yake katika ngeli za kihaya.

Tasnifu ya uzamili, Chuo Kikuu cha Dar es Salaam.

Ame, P. M. (2017). Athari za Kiisimu za Lahaja ya Kitumbatu katika Kiswahili Sanifu

Kinachotumika katika Shule za Tumbatu Zanzibar. Tasnifu ya Uzamili, Chuo Kikuu Huria cha Tanzania.

Barno, H. C. (2013). Muundo wa Kimofo-Sintaksia wa Kitenzi (Kt) cha Kinandi kwa Mtazamo wa Kiunzi cha Kanuni Finyu (Kkf). NordicJournalof African Studies, 22(4), 23-23.

Carolyne, O., Simala, I. K., \& John, K. M. (2017). Athari za uhamishaji wa sarufi ya Kiluo kwenye upatanisho wa sarufi ya Kiswahili. Kiswahili, 76(1).

Chomsky, N. (1981). Lectures on government and binding: The Pisa Lictures. Dordrecht: Foris.
Habwe, J. \& Karanja, P. (2004). Misingi ya sarufi ya Kiswahili. Nairobi: Jarada Phoenix Publishers Ltd.

Kapinga, M. C. (1983). Sarufi Maumbo ya Kiswahili sanifu. Taasisi ya Uchunguzi wa Kiswahili. Dar es Salaam, TZ: Chuo Kikuu cha Dar es Salaam.

Kihore, Y, M., Massamba, D. P. B. \& Msanjila, Y. P. (2004). Sarufi maumbo ya Kiswahili Sanifu (Samakisa) Sekondari na Vyuo. Dar es Salaam: Taasisi ya Uchunguzi wa Kiswahili, Chuo Kikuu cha Dar es Salaam.

Kithuku, M. (2012). Mofosintaksia ya ngeli ya $9 / 10$ (N/N). Nairobi, KE: Tasnifu ya Uzamili ya Chuo Kikuu cha Kenyatta.

Massamba, D. P. B. (2004). Kamusi ya isimu na falsafa ya lugha. Taasisi ya Uchunguzi wa Kiswahili, Chuo Kikuu cha Dar es Salaam.

Mathooko, P.M. (2004). Towards integrative perspective of linguistic accommodation: A case study of Kikamba and Kitharaka. (Unpublished $\mathrm{PhD}$ thesis). NAironi, KE: Kenyatta University.

Mgullu, R. S. (1999). Mtalaa wa isimu: fonetiki, fonolojia na mofolojia ya Kiswahili. Nairobi, KE: Longhorn Publishers.

Mramba, P. T. (2015). Uchunguzi wa athari za Kirombo katika jifunzaji wa kiswahili Vipengele vya kifonolojia na kimofolojia. Tasnifu ya Uzamili, Chuo Kikuu cha Dar es Salaam.

Mukuria, D. M. (1987). Mpangilio wa Ngeli: Ulinganishi wa Ngeli za Kiswahili sanifu na Kikikuyu cha Kiambu. Tasnifu ya Uzamili ya Chuo Kikuu cha Nairobi, Kenya.

Niyomugabo, C. (2016). Ufundishaji wa Kiswahili katika Shule za Rwanda: Historia, Tathmini ya Makosa, Matatizo na Mahitaji. Kiswahili, 79(1).

Ntawiyanga, S. (2015). Changamoto za kimofosintaksia miongoni mwa wanafunzi wa Kiswahili wa shule za upili wilayani Muhanga, Nchini Rwanda. Tasnifu ya uzamili ya Chuo Kikuu cha Kenyatta, Kenya.

22 | This work is licensed under a Creative Commons Attribution 4.0 International License. 
Nyanda, J. (2015). Athari za Lugha ya Kinyamwezi Katika Kujifunza Lugha ya Kiswahili. Tasnifu ya uzamili ya Chuo Kikuu Huria cha Tanzania.

Okumu, N. B. (2006) Tathmini ya mpango wa kisintaksia wa ngeli za nomino za Kiswahili: ufunzaji shule za Nairobi. Tasnifu ya uzamili ya Chuo Kikuu cha Kenyatta.

Otiende, M. A. (2013). Athari za Kimofofonolojia za Kiolusuba katika Matumizi ya Kiswahili sanifu kama Lugha ya Pili. Chuo Kikuu Cha Nairobi.

Shabani, A. N. \& R. (2015). Nadharia ya Utawala na Ufunganisho Inavyojihusisha na Aina za Vikundi Nomino katika Kiswahili, (2004), 1-8. 